\title{
ON THE MEASUREMENT OF GROWTH PROPERTIES OF ENTIRE AND MEROMORPHIC FUNCTIONS FOCUSING THEIR RELATIVE TYPE AND RELATIVE WEAK TYPE
}

\author{
Sanjib Kumar Datta and Tanmay Biswas
}

\begin{abstract}
The concepts of relative growth indicators such as relative order, relative type, relative weak type, etc. have widely been used to avoid comparing growths of entire and meromorphic functions just with exp functions. Using the notions of several relative growth indicators as mentioned earlier, in this paper we would like to find out the limits in terms of classical growth indicators (i.e. order, type, weak type etc.) in which the relative type, relative weak type, etc. of meromorphic functions with respect to entire functions should lie.
\end{abstract}

Keywords: Meromorphic function; Entire function; Relative order; Relative lower order; Relative type; Relative weak type

\section{Introduction, Definitions and Notations}

Let $\mathbb{C}$ be the set of all finite complex numbers. Let $f$ be an entire function defined on $\mathbb{C}$. The maximum modulus function corresponding to entire $f$ is defined as $M_{f}(r)=\max \{|f(z)|:|z|=r\}$. The order ( lower order) of an entire function $f$ is defined in terms of the growth of $f$ with respect to $\exp z$ function which is as follows:

$$
\begin{gathered}
\rho_{f}=\limsup _{r \rightarrow \infty} \frac{\log \log M_{f}(r)}{\log \log M_{\exp z}(r)}=\limsup _{r \rightarrow \infty} \frac{\log \log M_{f}(r)}{\log (r)} \\
\left(\lambda_{f}=\liminf _{r \rightarrow \infty} \frac{\log \log M_{f}(r)}{\log \log M_{\exp z}(r)}=\liminf _{r \rightarrow \infty} \frac{\log \log M_{f}(r)}{\log (r)}\right) .
\end{gathered}
$$

When $f$ is a meromorphic function, $M_{f}(r)$ cannot be defined as $f$ is not analytic. In this case one may define another function $T_{f}(r)$ known as Nevanlinna's

Received June 25, 2016; accepted September 29, 2016

2010 Mathematics Subject Classification. Primary 30D20; Secondary 30D30, 30D35 
Characteristic function of $f$, playing the same role as maximum modulus function in the following manner:

$$
T_{f}(r)=N_{f}(r)+m_{f}(r),
$$

where the function $N_{f}(r, a)\left(\bar{N}_{f}(r, a)\right)$ known as counting function of $a$-points (distinct $a$-points) of meromorphic $f$ is defined as

$$
\begin{gathered}
N_{f}(r, a)=\int_{0}^{r} \frac{n_{f}(t, a)-n_{f}(0, a)}{t} d t+\bar{n}_{f}(0, a) \log r \\
\left(\bar{N}_{f}(r, a)=\int_{0}^{r} \frac{\bar{n}_{f}(t, a)-\bar{n}_{f}(0, a)}{t} d t+\bar{n}_{f}(0, a) \log r\right),
\end{gathered}
$$

moreover we denote by $n_{f}(r, a)\left(\bar{n}_{f}(r, a)\right)$ the number of $a$-points (distinct $a$ points) of $f$ in $|z| \leq r$ and an $\infty$-point is a pole of $f$. In many occasions $N_{f}(r, \infty)$ and $\bar{N}_{f}(r, \infty)$ are denoted by $N_{f}(r)$ and $\bar{N}_{f}(r)$ respectively.

Also the function $m_{f}(r, \infty)$ alternatively denoted by $m_{f}(r)$ known as the proximity function of $f$ is defined as follows:

$$
m_{f}(r)=\frac{1}{2 \pi} \int_{0}^{2 \pi} \log +\left|f\left(r e^{i \theta}\right)\right| d \theta,
$$

where

$$
\log ^{+} x=\max (\log x, 0) \text { for all } x \geqslant 0 \text {. }
$$

Also we may denote $m\left(r, \frac{1}{f-a}\right)$ by $m_{f}(r, a)$.

If $f$ is an entire function, then the Nevanlinna's Characteristic function $T_{f}(r)$ of $f$ is defined as

$$
T_{f}(r)=m_{f}(r)
$$

Further, if $f$ is a non-constant entire then $T_{f}(r)$ is strictly increasing and continuous function of $r$. Also its inverse $T_{f}^{-1}:\left(T_{f}(0), \infty\right) \rightarrow(0, \infty)$ exist and is such that $\lim _{s \rightarrow \infty} T_{f}^{-1}(s)=\infty$. However, in case of meromorphic functions, the growth indicators such as order and lower order which are classical in complex analysis are defined in terms of their growths with respect to $\exp z$ function as the following:

$$
\begin{gathered}
\rho_{f}=\limsup _{r \rightarrow \infty} \frac{\log T_{f}(r)}{\log T_{\exp z}(r)}=\limsup _{r \rightarrow \infty} \frac{\log T_{f}(r)}{\log \left(\frac{r}{\pi}\right)}=\limsup _{r \rightarrow \infty} \frac{\log T_{f}(r)}{\log (r)+O(1)} \\
\left(\lambda_{f}=\liminf _{r \rightarrow \infty} \frac{\log T_{f}(r)}{\log T_{\exp z}(r)}=\liminf _{r \rightarrow \infty} \frac{\log T_{f}(r)}{\log \left(\frac{r}{\pi}\right)}=\liminf _{r \rightarrow \infty} \frac{\log T_{f}(r)}{\log (r)+O(1)}\right) .
\end{gathered}
$$


Next, we give the definitions of type and weak type of meromorphic functions which are also another type of classical growth indicators used for comparing the relative growth of two meromorphic functions having same non zero finite order with respect to another meromorphic function:

Definition 1.1. The type $\sigma_{f}$ and lower type $\bar{\sigma}_{f}$ of a meromorphic function $f$ are defined as

$$
\sigma_{f}=\limsup _{r \rightarrow \infty} \frac{T_{f}(r)}{r^{\rho_{f}}} \text { and } \bar{\sigma}_{f}=\liminf _{r \rightarrow \infty} \frac{T_{f}(r)}{r^{\rho_{f}}}, \quad 0<\rho_{f}<\infty .
$$

Datta and Jha [4] introduced the definition of weak type of a meromorphic function of finite positive lower order in the following way:

Definition 1.2. [4] The weak type $\tau_{f}$ and the growth indicator $\bar{\tau}_{f}$ of a meromorphic function $f$ of finite positive lower order $\lambda_{f}$ are defined by

$$
\bar{\tau}_{f}=\limsup _{r \rightarrow \infty} \frac{T_{f}(r)}{r^{\lambda_{f}}} \text { and } \tau_{f}=\liminf _{r \rightarrow \infty} \frac{T_{f}(r)}{r^{\lambda_{f}}}, 0<\lambda_{f}<\infty .
$$

Extending the notion of relative order as introduced by Bernal $\{[1],[2]\}$, Lahiri and Banerjee [7] gave the definition of relative order of a meromorphic function $f$ with respect to an entire function $g$, denoted by $\rho_{g}(f)$ as follows:

$$
\begin{aligned}
\rho_{g}(f) & =\inf \left\{\mu>0: T_{f}(r)<T_{g}\left(r^{\mu}\right) \text { for all sufficiently large } r\right\} \\
& =\limsup _{r \rightarrow \infty} \frac{\log T_{g}^{-1} T_{f}(r)}{\log r} .
\end{aligned}
$$

The definition coincides with the classical one [7] if $g(z)=\exp z$.

In the same way, one can define the relative lower order of a meromorphic function $f$ with respect to entire $g$ denoted by $\lambda_{g}(f)$ in the following manner :

$$
\lambda_{g}(f)=\liminf _{r \rightarrow \infty} \frac{\log T_{g}^{-1} T_{f}(r)}{\log r} .
$$

In the case of meromorphic functions, it therefore seems reasonable to define suitably the relative type and relative weak type of a meromorphic function with respect to an entire function to determine the relative growth of two meromorphic functions having same non zero finite relative order or relative lower order with respect to an entire function. Datta and Biswas [5] also gave such definitions of relative type and relative weak type of a meromorphic function $f$ with respect to an entire function $g$ which are as follows: 
Definition 1.3. [5] The relative type $\sigma_{g}(f)$ of a meromorphic function $f$ with respect to an entire function $g$ are defined as

$$
\sigma_{g}(f)=\limsup _{r \rightarrow \infty} \frac{T_{g}^{-1} T_{f}(r)}{r^{\rho_{g}(f)}}, \quad \text { where } 0<\rho_{g}(f)<\infty .
$$

Similarly, one can define the lower relative type $\bar{\sigma}_{g}(f)$ in the following way:

$$
\bar{\sigma}_{g}(f)=\liminf _{r \rightarrow \infty} \frac{T_{g}^{-1} T_{f}(r)}{r^{\rho_{g}(f)}}, \quad \text { where } 0<\rho_{g}(f)<\infty .
$$

Definition 1.4. [5] The relative weak type $\tau_{g}(f)$ of a meromorphic function $f$ with respect to an entire function $g$ with finite positive relative lower order $\lambda_{g}(f)$ is defined by

$$
\tau_{g}(f)=\liminf _{r \rightarrow \infty} \frac{T_{g}^{-1} T_{f}(r)}{r^{\lambda_{g}(f)}} .
$$

In a like manner, one can define the growth indicator $\bar{\tau}_{g}(f)$ of a meromorphic function $f$ with respect to an entire function $g$ with finite positive relative lower order $\lambda_{g}(f)$ as

$$
\bar{\tau}_{g}(f)=\limsup _{r \rightarrow \infty} \frac{T_{g}^{-1} T_{f}(r)}{r^{\lambda_{g}(f)}} .
$$

Considering $g=\exp z$ one may easily verify that Definition 1.3 and Definition 1.4 coincide with the classical definition of type (lower type) and weak type of a meromorphic function.

For entire and meromorphic functions, the notion of the growth indicators of its such as order, type and weak type are classical in complex analysis and during the past decades, several researchers have already been continued their studies in the area of comparative growth properties of entire and meromorphic functions in different directions using the growth indicator such as order, type and weak type. But at that time, the concept of relative order and consequently relative type and relative weak type of meromorphic function with respect to another entire function which have already been discussed above was mostly unknown to complex analysts and was not known for the technical advantage given by such notion which gives an idea to avoid comparing growth just with exp function to calculate order, type and weak type respectively. In the paper, we investigate some relative growth properties of entire and meromorphic functions with respect to another entire function on the basis of relative type and relative weak type. We use the standard notations and definitions of the theory of entire and meromorphic functions which are available in [6] and [9]. Hence we do not explain those in details.

\section{Lemma}

In this section we present a lemma due to Debnath et al. [3]: 
Lemma 2.1. [3] Let $f$ be a meromorphic function $0<\lambda_{f} \leq \rho_{f}<\infty$ and $g$ be an entire function with $0<\lambda_{g} \leq \rho_{g}<\infty$. Then

$$
\frac{\lambda_{f}}{\rho_{g}} \leq \lambda_{g}(f) \leq \min \left\{\frac{\lambda_{f}}{\lambda_{g}}, \frac{\rho_{f}}{\rho_{g}}\right\} \leq \max \left\{\frac{\lambda_{f}}{\lambda_{g}}, \frac{\rho_{f}}{\rho_{g}}\right\} \leq \rho_{g}(f) \leq \frac{\rho_{f}}{\lambda_{g}} .
$$

\section{Main Results}

In this section we state the main results of the paper.

Theorem 3.1. Let $f$ be a meromorphic function $0<\lambda_{f} \leq \rho_{f}<\infty$ and $g$ be an entire function with $0<\lambda_{g} \leq \rho_{g}<\infty$. Then

$$
\max \left\{\left[\frac{\bar{\sigma}_{f}}{\tau_{g}}\right]^{\frac{1}{\lambda_{g}}},\left[\frac{\sigma_{f}}{\bar{\tau}_{g}}\right]^{\frac{1}{\lambda_{g}}}\right\} \leq \sigma_{g}(f) \leq \min \left\{\left[\frac{\bar{\tau}_{f}}{\tau_{g}}\right]^{\frac{1}{\lambda_{g}}},\left[\frac{\sigma_{f}}{\bar{\sigma}_{g}}\right]^{\frac{1}{\rho_{g}}},\left[\frac{\bar{\tau}_{f}}{\overline{\bar{\sigma}}_{g}}\right]^{\frac{1}{\rho_{g}}}\right\}
$$

and

$\left[\frac{\bar{\sigma}_{f}}{\bar{\tau}_{g}}\right]^{\frac{1}{\lambda_{g}}} \leq \bar{\sigma}_{g}(f) \leq \min \left\{\left[\frac{\bar{\sigma}_{f}}{\bar{\sigma}_{g}}\right]^{\frac{1}{\rho_{g}}},\left[\frac{\sigma_{f}}{\sigma_{g}}\right]^{\frac{1}{\rho_{g}}},\left[\frac{\tau_{f}}{\tau_{g}}\right]^{\frac{1}{\lambda_{g}}},\left[\frac{\bar{\tau}_{f}}{\bar{\tau}_{g}}\right]^{\frac{1}{\lambda_{g}}},\left[\frac{\bar{\tau}_{f}}{\sigma_{g}}\right]^{\frac{1}{\rho_{g}}},\left[\left[\frac{\tau_{f}}{\bar{\sigma}_{g}}\right]^{\frac{1}{\rho_{g}}}\right\}\right.$

Proof. From the definitions of $\sigma_{f}$ and $\bar{\sigma}_{f}$, we have for all sufficiently large values of $r$ that

$$
\begin{aligned}
& T_{f}(r) \leq\left(\sigma_{f}+\varepsilon\right) \cdot r^{\rho_{f}}, \\
& T_{f}(r) \geq\left(\bar{\sigma}_{f}-\varepsilon\right) \cdot r^{\rho_{f}}
\end{aligned}
$$

and also for a sequence of values of $r$ tending to infinity we get that

$$
\begin{aligned}
& T_{f}(r) \geq\left(\sigma_{f}-\varepsilon\right) \cdot r^{\rho_{f}}, \\
& T_{f}(r) \leq\left(\bar{\sigma}_{f}+\varepsilon\right) \cdot r^{\rho_{f}} .
\end{aligned}
$$

Similarly from the definitions of $\sigma_{g}$ and $\bar{\sigma}_{g}$, it follows for all sufficiently large values of $r$ that

$$
\begin{aligned}
& T_{g}(r) \leq\left(\sigma_{g}+\varepsilon\right) \cdot r^{\rho_{g}} \text { i.e., } r \leq T_{g}^{-1}\left[\left(\sigma_{g}+\varepsilon\right) \cdot r^{\rho_{g}}\right] \text { i.e., } T_{g}^{-1}(r) \geq\left[\left(\frac{r}{\left(\sigma_{g}+\varepsilon\right)}\right)^{\frac{1}{\rho_{g}}}\right], \\
& T_{g}(r) \geq\left(\bar{\sigma}_{g}-\varepsilon\right) \cdot r^{\rho_{g}} \text { i.e., } r \geq T_{g}^{-1}\left[\left(\bar{\sigma}_{g}-\varepsilon\right) \cdot r^{\rho_{g}}\right] \text { i.e., } T_{g}^{-1}(r) \leq\left[\left(\frac{r}{\left(\bar{\sigma}_{g}-\varepsilon\right)}\right)^{\frac{1}{\rho_{g}}}\right]
\end{aligned}
$$


and for a sequence of values of $r$ tending to infinity, we obtain that $T_{g}(r) \geq\left(\sigma_{g}-\varepsilon\right) \cdot r^{\rho_{g}} i . e ., r \geq T_{g}^{-1}\left[\left(\sigma_{g}-\varepsilon\right) \cdot r^{\rho_{g}}\right]$ i.e., $T_{g}^{-1}(r) \leq\left[\left(\frac{r}{\left(\sigma_{g}-\varepsilon\right)}\right)^{\frac{1}{\rho_{g}}}\right]$, $T_{g}(r) \leq\left(\bar{\sigma}_{g}+\varepsilon\right) \cdot r^{\rho_{g}}$ i.e., $r \leq T_{g}^{-1}\left[\left(\bar{\sigma}_{g}+\varepsilon\right) \cdot r^{\rho_{g}}\right]$ i.e., $T_{g}^{-1}(r) \geq\left[\left(\frac{r}{\left(\bar{\sigma}_{g}-\varepsilon\right)}\right)^{\frac{1}{\rho_{g}}}\right]$.

Further from the definitions of $\bar{\tau}_{f}$ and $\tau_{f}$, it follows for all sufficiently large values of $r$ that

$$
\begin{aligned}
& T_{f}(r) \leq\left(\bar{\tau}_{f}+\varepsilon\right) \cdot r^{\lambda_{f}}, \\
& T_{f}(r) \geq\left(\tau_{f}-\varepsilon\right) \cdot r^{\lambda_{f}}
\end{aligned}
$$

and also for a sequence of values of $r$ tending to infinity, we get that

$$
\begin{aligned}
& T_{f}(r) \geq\left(\bar{\tau}_{f}-\varepsilon\right) \cdot r^{\lambda_{f}}, \\
& T_{f}(r) \leq\left(\tau_{f}+\varepsilon\right) \cdot r^{\lambda_{f}} .
\end{aligned}
$$

Also from the definitions of $\bar{\tau}_{g}$ and $\tau_{g}$, we obtain for all sufficiently large values of $r$ that

$T_{g}(r) \leq\left(\bar{\tau}_{g}+\varepsilon\right) \cdot r^{\lambda_{g}} i . e ., r \leq T_{g}^{-1}\left[\left(\bar{\tau}_{g}+\varepsilon\right) \cdot r^{\lambda_{g}}\right]$ i.e., $T_{g}^{-1}(r) \geq\left[\left(\frac{r}{\left(\bar{\tau}_{g}+\varepsilon\right)}\right)^{\frac{1}{\lambda_{g}}}\right]$,

$$
T_{g}(r) \geq\left(\tau_{g}-\varepsilon\right) \cdot r^{\lambda_{g}} \text { i.e., } r \geq T_{g}^{-1}\left[\left(\tau_{g}-\varepsilon\right) \cdot r^{\lambda_{g}}\right] \text { i.e., } T_{g}^{-1}(r) \leq\left[\left(\frac{r}{\left(\tau_{g}-\varepsilon\right)}\right)^{\frac{1}{\lambda_{g}}}\right]
$$

and for a sequence of values of $r$ tending to infinity, we obtain that

$$
\begin{aligned}
& T_{g}(r) \geq(\bar{\tau}-\varepsilon) \cdot r^{\lambda_{g}} i . e ., r \geq T_{g}^{-1}\left[\left(\bar{\tau}_{g}-\varepsilon\right) \cdot r^{\lambda_{g}}\right] \text { i.e., } T_{g}^{-1}(r) \leq\left[\left(\frac{r}{\left(\bar{\tau}_{g}-\varepsilon\right)}\right)^{\frac{1}{\lambda_{g}}}\right], \\
& T_{g}(r) \leq\left(\tau_{g}+\varepsilon\right) \cdot r^{\lambda_{g}} \text { i.e., } r \leq T_{g}^{-1}\left[\left(\tau_{g}+\varepsilon\right) \cdot r^{\lambda_{g}}\right] \text { i.e., } T_{g}^{-1}(r) \geq\left[\left(\frac{r}{\left(\tau_{g}-\varepsilon\right)}\right)^{\frac{1}{\lambda_{g}}}\right] .
\end{aligned}
$$

Now from (3.3) and in view of (3.9), we get for a sequence of values of $r$ tending to infinity that

$$
\begin{gathered}
T_{g}^{-1} T_{f}(r) \geq T_{g}^{-1}\left[\left(\sigma_{f}-\varepsilon\right) \cdot r^{\rho_{f}}\right] \\
\text { i.e., } T_{g}^{-1} T_{f}(r) \geq\left(\frac{\left(\sigma_{f}-\varepsilon\right) \cdot r^{\rho_{f}}}{\left(\bar{\tau}_{g}+\varepsilon\right)}\right)^{\frac{1}{\lambda_{g}}}
\end{gathered}
$$


On the Measurement of Growth Properties of Entire and Meromorphic Functions 1017

$$
\text { i.e., } T_{g}^{-1} T_{f}(r) \geq\left[\frac{\left(\sigma_{f}-\varepsilon\right)}{\left(\bar{\tau}_{g}+\varepsilon\right)}\right]^{\frac{1}{\lambda_{g}}} \cdot r^{\frac{\rho_{f}}{\lambda_{g}}} i . e ., \frac{T_{g}^{-1} T_{f}(r)}{r^{\frac{\rho_{f}}{\lambda_{g}}}} \geq\left[\frac{\left(\sigma_{f}-\varepsilon\right)}{\left(\bar{\tau}_{g}+\varepsilon\right)}\right]^{\frac{1}{\lambda_{g}}} \text {. }
$$

Since in view of Lemma 2.1, $\frac{\rho_{f}}{\lambda_{g}} \geq \rho_{g}(f)$ and as $\varepsilon(>0)$ is arbitrary, therefore it follows from above that

$$
\limsup _{r \rightarrow \infty} \frac{T_{g}^{-1} T_{f}(r)}{(r)^{\rho_{g}(f)}} \geq\left[\frac{\sigma_{f}}{\bar{\tau}_{g}}\right]^{\frac{1}{\lambda_{g}}} \text { i.e., } \sigma_{g}(f) \geq\left[\frac{\sigma_{f}}{\bar{\tau}_{g}}\right]^{\frac{1}{\lambda_{g}}} .
$$

Similarly from (3.2) and in view of (3.9), it follows for a sequence of values of $r$ tending to infinity that

$$
\begin{gathered}
T_{g}^{-1} T_{f}(r) \geq T_{g}^{-1}\left[\left(\bar{\sigma}_{f}-\varepsilon\right) \cdot r^{\rho_{f}}\right] \\
\text { i.e., } T_{g}^{-1} T_{f}(r) \geq\left(\frac{\left(\bar{\sigma}_{f}-\varepsilon\right) \cdot r^{\rho_{f}}}{\left(\tau_{g}-\varepsilon\right)}\right)^{\frac{1}{\lambda_{g}}} \\
\text { i.e., } T_{g}^{-1} T_{f}(r) \geq\left[\frac{\left(\bar{\sigma}_{f}-\varepsilon\right)}{\left(\tau_{g}+\varepsilon\right)}\right]^{\frac{1}{\lambda_{g}}} \cdot r^{\frac{\rho_{f}}{\lambda_{g}}} i . e ., \frac{T_{g}^{-1} T_{f}(r)}{r^{\frac{\rho_{f}}{\lambda_{g}}}} \geq\left[\frac{\left(\bar{\sigma}_{f}-\varepsilon\right)}{\left(\tau_{g}+\varepsilon\right)}\right]^{\frac{1}{\lambda_{g}}} .
\end{gathered}
$$

Since in view of Lemma 2.1, it follows that $\frac{\rho_{f}}{\lambda_{g}} \geq \rho_{g}(f)$. Also $\varepsilon(>0)$ is arbitrary. So we get from above that

$$
\limsup _{r \rightarrow \infty} \frac{T_{g}^{-1} T_{f}(r)}{r^{\rho_{g}(f)}} \geq\left[\frac{\bar{\sigma}_{f}}{\tau_{g}}\right]^{\frac{1}{\lambda_{g}}} \text { i.e., } \sigma_{g}(f) \geq\left[\frac{\bar{\sigma}_{f}}{\tau_{g}}\right]^{\frac{1}{\lambda_{g}}} .
$$

Again in view of (3.9), we have from (3.5) for all sufficiently large values of $r$ that

$$
\begin{gathered}
T_{g}^{-1} T_{f}(r) \leq T_{g}^{-1}\left[\left(\bar{\tau}_{f}+\varepsilon\right) \cdot r^{\lambda_{f}}\right] \\
\text { i.e., } T_{g}^{-1} T_{f}(r) \leq\left(\frac{\left(\bar{\tau}_{f}+\varepsilon\right) \cdot r^{\lambda_{f}}}{\left(\tau_{g}-\varepsilon\right)}\right)^{\frac{1}{\lambda_{g}}} \\
\text { i.e., } T_{g}^{-1} T_{f}(r) \leq\left[\frac{\left(\bar{\tau}_{f}+\varepsilon\right)}{\left(\tau_{g}-\varepsilon\right)}\right]^{\frac{1}{\lambda_{g}}} \cdot r^{\frac{\lambda_{f}}{\lambda_{g}}} i . e ., \frac{T_{g}^{-1} T_{f}(r)}{r^{\frac{\lambda_{f}}{\lambda_{g}}}} \leq\left[\frac{\left(\bar{\tau}_{f}+\varepsilon\right)}{\left(\tau_{g}-\varepsilon\right)}\right]^{\frac{1}{\lambda_{g}}} .
\end{gathered}
$$

Since in view of Lemma 2.1, we get that $\frac{\lambda_{f}}{\lambda_{g}} \leq \rho_{g}(f)$ and as $\varepsilon(>0)$ is arbitrary. Therefore it follows from above that

$$
\limsup _{r \rightarrow \infty} \frac{T_{g}^{-1} T_{f}(r)}{r^{\rho_{g}(f)}} \leq\left[\frac{\bar{\tau}_{f}}{\tau_{g}}\right]^{\frac{1}{\lambda_{g}}} \text { i.e., } \sigma_{g}(f) \leq\left[\frac{\bar{\tau}_{f}}{\tau_{g}}\right]^{\frac{1}{\lambda_{g}}} \text {. }
$$

Again in view of (3.5), we have from (3.1) for all sufficiently large values of $r$ that

$$
T_{g}^{-1} T_{f}(r) \leq T_{g}^{-1}\left[\left(\sigma_{f}+\varepsilon\right) \cdot r^{\rho_{f}}\right]
$$




$$
\begin{gathered}
\text { i.e., } T_{g}^{-1} T_{f}(r) \leq\left(\frac{\left(\sigma_{f}+\varepsilon\right) \cdot r^{\rho_{f}}}{\left(\bar{\sigma}_{g}-\varepsilon\right)}\right)^{\frac{1}{\rho_{g}}} \\
\text { i.e., } T_{g}^{-1} T_{f}(r) \leq\left[\frac{\left(\sigma_{f}+\varepsilon\right)}{\left(\bar{\sigma}_{g}-\varepsilon\right)}\right]^{\frac{1}{\rho_{g}}} \cdot r^{\frac{\rho_{f}}{\rho_{g}}} \text { i.e., } \frac{T_{g}^{-1} T_{f}(r)}{r^{\frac{\rho_{f}}{\rho_{g}}}} \leq\left[\frac{\left(\sigma_{f}+\varepsilon\right)}{\left(\bar{\sigma}_{g}-\varepsilon\right)}\right]^{\frac{1}{\rho_{g}}} .
\end{gathered}
$$

As in view of Lemma 2.1, it follows that $\frac{\rho_{f}}{\rho_{g}} \leq \rho_{g}(f)$. Since $\varepsilon(>0)$ is arbitrary, we get from (3.9) that

$$
\begin{aligned}
\limsup _{r \rightarrow \infty} \frac{T_{g}^{-1} T_{f}(r)}{r^{\rho_{g}(f)}} & \leq\left[\frac{\sigma_{f}}{\bar{\sigma}_{g}}\right]^{\frac{1}{\rho_{g}}} \\
\text { i.e., } \sigma_{g}(f) & \leq\left[\frac{\sigma_{f}}{\bar{\sigma}_{g}}\right]^{\frac{1}{\rho_{g}}} .
\end{aligned}
$$

Further in view of (3.5), we have from (3.5) for all sufficiently large values of $r$ that

$$
\begin{gathered}
T_{g}^{-1} T_{f}(r) \leq T_{g}^{-1}\left[\left(\bar{\tau}_{f}+\varepsilon\right) \cdot r^{\lambda_{f}}\right] \\
\text { i.e., } T_{g}^{-1} T_{f}(r) \leq\left(\frac{\left(\bar{\tau}_{f}+\varepsilon\right) \cdot r^{\lambda_{f}}}{\left(\bar{\sigma}_{g}-\varepsilon\right)}\right)^{\frac{1}{\rho_{g}}} \\
\text { i.e., } T_{g}^{-1} T_{f}(r) \leq\left[\frac{\left(\bar{\tau}_{f}+\varepsilon\right)}{\left(\bar{\sigma}_{g}-\varepsilon\right)}\right]^{\frac{1}{\rho_{g}}} \cdot r^{\frac{\lambda_{f}}{\rho_{g}}} \\
\text { i.e., } \frac{T_{g}^{-1} T_{f}(r)}{r^{\frac{\lambda_{f}}{\rho_{g}}}} \leq\left[\frac{\left(\bar{\tau}_{f}+\varepsilon\right)}{\left(\bar{\sigma}_{g}-\varepsilon\right)}\right]^{\frac{1}{\rho_{g}}} .
\end{gathered}
$$

Since in view of Lemma 2.1, we get that $\frac{\lambda_{f}}{\rho_{g}} \leq \rho_{g}(f)$ and as $\varepsilon(>0)$ is arbitrary. Therefore it follows from above that

$$
\begin{aligned}
\limsup _{r \rightarrow \infty} \frac{T_{g}^{-1} T_{f}(r)}{r^{\rho_{g}(f)}} & \leq\left[\frac{\bar{\tau}_{f}}{\bar{\sigma}_{g}}\right]^{\frac{1}{\rho_{g}}} \\
\text { i.e., } \sigma_{g}(f) & \leq\left[\frac{\bar{\tau}_{f}}{\bar{\sigma}_{g}}\right]^{\frac{1}{\rho_{g}}} .
\end{aligned}
$$

Thus the first part of the theorem follows from $(3.9),(3.9),(3.9),(3.9)$ and (3.9).

Further from (3.2) and in view of (3.9), we get for all sufficiently large values of $r$ that

$$
\begin{gathered}
T_{g}^{-1} T_{f}(r) \geq T_{g}^{-1}\left[\left(\bar{\sigma}_{f}-\varepsilon\right) \cdot r^{\rho_{f}}\right] \\
\text { i.e., } T_{g}^{-1} T_{f}(r) \geq\left(\frac{\left(\bar{\sigma}_{f}-\varepsilon\right) \cdot r^{\rho_{f}}}{\left(\bar{\tau}_{g}+\varepsilon\right)}\right)^{\frac{1}{\lambda_{g}}} \\
\text { i.e., } T_{g}^{-1} T_{f}(r) \geq\left[\frac{\left(\bar{\sigma}_{f}-\varepsilon\right)}{\left(\bar{\tau}_{g}+\varepsilon\right)}\right]^{\frac{1}{\lambda_{g}}} \cdot r^{\frac{\rho_{f}}{\lambda_{g}}} \\
\text { i.e., } \frac{T_{g}^{-1} T_{f}(r)}{r^{\frac{\rho_{f}}{\lambda_{g}}}} \geq\left[\frac{\left(\bar{\sigma}_{f}-\varepsilon\right)}{\left(\bar{\tau}_{g}+\varepsilon\right)}\right]^{\frac{1}{\lambda_{g}}} .
\end{gathered}
$$


Now in view of Lemma 2.1, it follows that $\frac{\rho_{f}}{\lambda_{g}} \geq \rho_{g}(f)$. Since $\varepsilon(>0)$ is arbitrary, we get from above that

$$
\begin{aligned}
\liminf _{r \rightarrow \infty} \frac{T_{g}^{-1} T_{f}(r)}{r^{\rho_{g}(f)}} & \geq\left[\frac{\bar{\sigma}_{f}}{\bar{\tau}_{g}}\right]^{\frac{1}{\lambda_{g}}} \\
\text { i.e., } \bar{\sigma}_{g}(f) & \geq\left[\frac{\bar{\sigma}_{f}}{\bar{\tau}_{g}}\right]^{\frac{1}{\lambda_{g}}} .
\end{aligned}
$$

Also in view of (3.5), we get from (3.1) for a sequence of values of $r$ tending to infinity that

$$
\begin{gathered}
T_{g}^{-1} T_{f}(r) \leq T_{g}^{-1}\left[\left(\sigma_{f}+\varepsilon\right) \cdot r^{\rho_{f}}\right] \\
\text { i.e., } T_{g}^{-1} T_{f}(r) \leq\left(\frac{\left(\sigma_{f}+\varepsilon\right) \cdot r^{\rho_{f}}}{\left(\sigma_{g}-\varepsilon\right)}\right)^{\frac{1}{\rho_{g}}} \\
\text { i.e., } T_{g}^{-1} T_{f}(r) \leq\left[\frac{\left(\sigma_{f}+\varepsilon\right)}{\left(\sigma_{g}-\varepsilon\right)}\right]^{\frac{1}{\rho_{g}}} \cdot r^{\frac{\rho_{f}}{\rho_{g}}} \\
\text { i.e., } \frac{T_{g}^{-1} T_{f}(r)}{r^{\frac{\rho_{f}}{\rho_{g}}}} \leq\left[\frac{\left(\sigma_{f}+\varepsilon\right)}{\left(\sigma_{g}-\varepsilon\right)}\right]^{\frac{1}{\rho_{g}(m, p)}} .
\end{gathered}
$$

Again in view of Lemma 2.1, $\frac{\rho_{f}}{\rho_{g}} \leq \rho_{g}(f)$ and $\varepsilon(>0)$ is arbitrary, so we get from (3.9) that

$$
\begin{aligned}
\liminf _{r \rightarrow \infty} \frac{T_{g}^{-1} T_{f}(r)}{r^{\rho_{g}(f)}} & \leq\left[\frac{\sigma_{f}}{\sigma_{g}}\right]^{\frac{1}{\rho_{g}}} \\
\text { i.e., } \bar{\sigma}_{g}(f) & \leq\left[\frac{\sigma_{f}}{\sigma_{g}}\right]^{\frac{1}{\rho_{g}}} .
\end{aligned}
$$

Likewise from (3.4) and in view of (3.5), it follows for a sequence of values of $r$ tending to infinity that

$$
\begin{gathered}
T_{g}^{-1} T_{f}(r) \leq T_{g}^{-1}\left[\left(\bar{\sigma}_{f}+\varepsilon\right) \cdot r^{\rho_{f}}\right] \\
\text { i.e., } T_{g}^{-1} T_{f}(r) \leq\left(\frac{\left(\bar{\sigma}_{f}+\varepsilon\right) \cdot r^{\rho_{f}}}{\left(\bar{\sigma}_{g}-\varepsilon\right)}\right)^{\frac{1}{\rho_{g}}} \\
\text { i.e., } T_{g}^{-1} T_{f}(r) \leq\left[\frac{\left(\bar{\sigma}_{f}+\varepsilon\right)}{\left(\bar{\sigma}_{g}-\varepsilon\right)}\right]^{\frac{1}{\rho_{g}}} \cdot r^{\frac{\rho_{f}}{\rho_{g}}} \\
\text { i.e., } \frac{T_{g}^{-1} T_{f}(r)}{r^{\frac{\rho_{f}}{\rho_{g}}}} \leq\left[\frac{\left(\bar{\sigma}_{f}+\varepsilon\right)}{\left(\bar{\sigma}_{g}-\varepsilon\right)}\right]^{\frac{1}{\rho_{g}}} .
\end{gathered}
$$

Analogously, we get from (3.9) that

$$
\begin{aligned}
\liminf _{r \rightarrow \infty} \frac{T_{g}^{-1} T_{f}(r)}{r^{\rho_{g}(f)}} & \leq\left[\frac{\bar{\sigma}_{f}}{\bar{\sigma}_{g}}\right]^{\frac{1}{\rho_{g}}} \\
\text { i.e., } & \bar{\sigma}_{g}(f) \leq\left[\frac{\bar{\sigma}_{f}}{\bar{\sigma}_{g}}\right]^{\frac{1}{\rho_{g}}},
\end{aligned}
$$


since in view of Lemma 2.1, $\frac{\rho_{f}}{\rho_{g}} \leq \rho_{g}(f)$ and $\varepsilon(>0)$ is arbitrary.

Further in view of (3.9), we get from (3.5) for a sequence of values of $r$ tending to infinity that

$$
\begin{gathered}
T_{g}^{-1} T_{f}(r) \leq T_{g}^{-1}\left[\left(\bar{\tau}_{f}+\varepsilon\right) \cdot r^{\lambda_{f}}\right] \\
\text { i.e., } T_{g}^{-1} T_{f}(r) \leq\left(\frac{\left(\bar{\tau}_{f}+\varepsilon\right) \cdot r^{\lambda_{f}}}{\left(\bar{\tau}_{g}-\varepsilon\right)}\right)^{\frac{1}{\lambda_{g}}} \\
\text { i.e., } T_{g}^{-1} T_{f}(r) \leq\left[\frac{\left(\bar{\tau}_{f}+\varepsilon\right)}{\left(\bar{\tau}_{g}-\varepsilon\right)}\right]^{\frac{1}{\lambda_{g}}} \cdot r^{\frac{\lambda_{f}}{\lambda_{g}}} \\
\text { i.e., } \frac{T_{g}^{-1} T_{f}(r)}{r^{\frac{\lambda_{f}}{\lambda_{g}}}} \leq\left[\frac{\left(\bar{\tau}_{f}+\varepsilon\right)}{\left(\bar{\tau}_{g}-\varepsilon\right)}\right]^{\frac{1}{\lambda_{g}}} .
\end{gathered}
$$

As in view of Lemma 2.1, we get that $\frac{\lambda_{f}}{\lambda_{g}} \leq \rho_{g}(f)$ and as $\varepsilon(>0)$ is arbitrary. Therefore it follows from above that

$$
\begin{aligned}
\liminf _{r \rightarrow \infty} \frac{T_{g}^{-1} T_{f}(r)}{r^{\rho_{g}(f)}} & \leq\left[\frac{\bar{\tau}_{f}}{\bar{\tau}_{g}}\right]^{\frac{1}{\lambda_{g}}} \\
\text { i.e., } \bar{\sigma}_{g}(f) & \leq\left[\frac{\bar{\tau}_{f}}{\bar{\tau}_{g}}\right]^{\frac{1}{\lambda_{g}}} .
\end{aligned}
$$

Similarly from (3.8) and in view of (3.9), it follows for a sequence of values of $r$ tending to infinity that

$$
\begin{gathered}
T_{g}^{-1} T_{f}(r) \leq T_{g}^{-1}\left[\left(\tau_{f}+\varepsilon\right) \cdot r^{\lambda_{f}}\right] \\
\text { i.e., } T_{g}^{-1} T_{f}(r) \leq\left(\frac{\left(\tau_{f}+\varepsilon\right) \cdot r^{\lambda_{f}}}{\left(\tau_{g}-\varepsilon\right)}\right)^{\frac{1}{\lambda_{g}}} \\
\text { i.e., } T_{g}^{-1} T_{f}(r) \leq\left[\frac{\left(\tau_{f}+\varepsilon\right)}{\left(\tau_{g}-\varepsilon\right)}\right]^{\frac{1}{\lambda_{g}}} \cdot r^{\frac{\lambda_{f}}{\lambda_{g}}} \\
\text { i.e., } \frac{T_{g}^{-1} T_{f}(r)}{r^{\frac{\lambda_{f}}{\lambda_{g}}}} \leq\left[\frac{\left(\tau_{f}+\varepsilon\right)}{\left(\tau_{g}-\varepsilon\right)}\right]^{\frac{1}{\lambda_{g}}} .
\end{gathered}
$$

Also in view of Lemma 2.1, we get that $\frac{\lambda_{f}}{\lambda_{g}} \leq \rho_{g}(f)$ and as $\varepsilon(>0)$ is arbitrary, therefore it follows from above that

$$
\begin{aligned}
\liminf _{r \rightarrow \infty} \frac{T_{g}^{-1} T_{f}(r)}{r^{\rho_{g}(f)}} & \leq\left[\frac{\tau_{f}}{\tau_{g}}\right]^{\frac{1}{\lambda_{g}}} \\
\text { i.e., } & \bar{\sigma}_{g}(f) \leq\left[\frac{\tau_{f}}{\tau_{g}}\right]^{\frac{1}{\lambda_{g}}} .
\end{aligned}
$$

Again in view of (3.5), we get from (3.5) for a sequence of values of $r$ tending to infinity that

$$
T_{g}^{-1} T_{f}(r) \leq T_{g}^{-1}\left[\left(\bar{\tau}_{f}+\varepsilon\right) \cdot r^{\lambda_{f}}\right]
$$


On the Measurement of Growth Properties of Entire and Meromorphic Functions 1021

$$
\begin{aligned}
& \text { i.e., } T_{g}^{-1} T_{f}(r) \leq\left(\frac{\left(\bar{\tau}_{f}+\varepsilon\right) \cdot r^{\lambda_{f}}}{\left(\sigma_{g}-\varepsilon\right)}\right)^{\frac{1}{\rho_{g}}} \\
& \text { i.e., } T_{g}^{-1} T_{f}(r) \leq\left[\frac{\left(\bar{\tau}_{f}+\varepsilon\right)}{\left(\sigma_{g}-\varepsilon\right)}\right]^{\frac{1}{\rho_{g}}} \cdot r^{\frac{\lambda_{f}}{\rho_{g}}} \\
& \text { i.e., } \frac{T_{g}^{-1} T_{f}(r)}{r^{\frac{\lambda_{f}}{\rho_{g}}}} \leq\left[\frac{\left(\bar{\tau}_{f}+\varepsilon\right)}{\left(\sigma_{g}-\varepsilon\right)}\right]^{\frac{1}{\rho_{g}}} .
\end{aligned}
$$

Since in view of Lemma 2.1, we get that $\frac{\lambda_{f}}{\rho_{g}} \leq \rho_{g}(f)$ and as $\varepsilon(>0)$ is arbitrary, so it follows from above that

$$
\begin{aligned}
\liminf _{r \rightarrow \infty} \frac{T_{g}^{-1} T_{f}(r)}{r^{\rho_{g}(f)}} & \leq\left[\frac{\bar{\tau}_{f}}{\sigma_{g}}\right]^{\frac{1}{\rho_{g}}} \\
\text { i.e., } \bar{\sigma}_{g}(f) & \leq\left[\frac{\bar{\tau}_{f}}{\sigma_{g}}\right]^{\frac{1}{\rho_{g}}} .
\end{aligned}
$$

Similarly from (3.8) and in view of (3.5), it follows for a sequence of values of $r$ tending to infinity that

$$
\begin{gathered}
T_{g}^{-1} T_{f}(r) \leq T_{g}^{-1}\left[\left(\tau_{f}+\varepsilon\right) \cdot r^{\lambda_{f}}\right] \\
\text { i.e., } T_{g}^{-1} T_{f}(r) \leq\left(\frac{\left(\tau_{f}+\varepsilon\right) \cdot r^{\lambda_{f}}}{\left(\bar{\sigma}_{g}-\varepsilon\right)}\right)^{\frac{1}{\rho_{g}}} \\
\text { i.e., } T_{g}^{-1} T_{f}(r) \leq\left[\frac{\left(\tau_{f}+\varepsilon\right)}{\left(\bar{\sigma}_{g}-\varepsilon\right)}\right]^{\frac{1}{\rho_{g}}} \cdot r^{\frac{\lambda_{f}}{\rho_{g}}} \\
\text { i.e., } \frac{T_{g}^{-1} T_{f}(r)}{r^{\frac{\lambda_{f}}{\rho_{g}}}} \leq\left[\frac{\left(\tau_{f}+\varepsilon\right)}{\left(\bar{\sigma}_{g}-\varepsilon\right)}\right]^{\frac{1}{\rho_{g}}} .
\end{gathered}
$$

As in view of Lemma 2.1, we get that $\frac{\lambda_{f}}{\rho_{g}} \leq \rho_{g}(f)$. Also as $\varepsilon(>0)$ is arbitrary, therefore it follows from above that

$$
\begin{aligned}
\liminf _{r \rightarrow \infty} \frac{T_{g}^{-1} T_{f}(r)}{r^{\rho_{g}(f)}} & \leq\left[\frac{\tau_{f}}{\bar{\sigma}_{g}}\right]^{\frac{1}{\rho_{g}}} \\
\text { i.e., } \quad \bar{\sigma}_{g}(f) & \leq\left[\frac{\tau_{f}}{\bar{\sigma}_{g}}\right]^{\frac{1}{\rho_{g}}} .
\end{aligned}
$$

Hence the second part of the theorem follows from $(3.9),(3.9),(3.9),(3.9),(3.9)$, (3.9) and (3.9).

Theorem 3.2. Let $f$ be a meromorphic function $0<\lambda_{f} \leq \rho_{f}<\infty$ and $g$ be an entire function with $0<\lambda_{g} \leq \rho_{g}<\infty$. Then

$\max \left\{\left[\frac{\bar{\tau}_{f}}{\bar{\tau}_{g}}\right]^{\frac{1}{\lambda_{g}}},\left[\frac{\tau_{f}}{\tau_{g}}\right]^{\frac{1}{\lambda_{g}}},\left[\frac{\bar{\sigma}_{f}}{\bar{\sigma}_{g}}\right]^{\frac{1}{\rho_{g}}},\left[\frac{\sigma_{f}}{\sigma_{g}}\right]^{\frac{1}{\rho_{g}}},\left[\frac{\sigma_{f}}{\bar{\tau}_{g}}\right]^{\frac{1}{\lambda_{g}}},\left[\frac{\bar{\sigma}_{f}}{\tau_{g}}\right]^{\frac{1}{\lambda_{g}}}\right\} \leq \bar{\tau}_{g}(f) \leq\left[\frac{\bar{\tau}_{f}}{\bar{\sigma}_{g}}\right]^{\frac{1}{\rho_{g}}}$ 
and

$$
\max \left\{\left[\frac{\bar{\sigma}_{f}}{\sigma_{g}}\right]^{\frac{1}{\rho_{g}}},\left[\frac{\tau_{f}}{\bar{\tau}_{g}}\right]^{\frac{1}{\lambda_{g}}},\left[\frac{\bar{\sigma}_{f}}{\bar{\tau}_{g}}\right]^{\frac{1}{\lambda_{g}}}\right\} \leq \tau_{g}(f) \leq \min \left\{\left[\frac{\tau_{f}}{\bar{\sigma}_{g}}\right]^{\frac{1}{\rho_{g}}},\left[\frac{\bar{\tau}_{f}}{\sigma_{g}}\right]^{\frac{1}{\rho_{g}}}\right\} .
$$

Proof. We obtain from (3.7) and (3.9), for a sequence of values of $r$ tending to infinity that

$$
\begin{gathered}
T_{g}^{-1} T_{f}(r) \geq T_{g}^{-1}\left[\left(\bar{\tau}_{f}-\varepsilon\right) \cdot r^{\lambda_{f}}\right] \\
\text { i.e., } T_{g}^{-1} T_{f}(r) \geq\left(\frac{\left(\bar{\tau}_{f}-\varepsilon\right) \cdot r^{\lambda_{f}}}{\left(\bar{\tau}_{g}+\varepsilon\right)}\right)^{\frac{1}{\lambda_{g}}} \\
\text { i.e., } T_{g}^{-1} T_{f}(r) \geq\left[\frac{\left(\bar{\tau}_{f}-\varepsilon\right)}{\left(\bar{\tau}_{g}+\varepsilon\right)}\right]^{\frac{1}{\lambda_{g}}} \cdot r^{\frac{\lambda_{f}}{\lambda_{g}}} \\
\text { i.e., } \frac{T_{g}^{-1} T_{f}(r)}{r^{\frac{\lambda_{f}}{\lambda_{g}}}} \geq\left[\frac{\left(\bar{\tau}_{f}-\varepsilon\right)}{\left(\bar{\tau}_{g}+\varepsilon\right)}\right]^{\frac{1}{\lambda_{g}}} .
\end{gathered}
$$

Since in view of Lemma 2.1, we get that $\frac{\lambda_{f}}{\lambda_{g}} \geq \lambda_{g}(f)$ and as $\varepsilon(>0)$ is arbitrary, therefore it follows from above that

$$
\begin{aligned}
\limsup _{r \rightarrow \infty} \frac{T_{g}^{-1} T_{f}(r)}{r^{\lambda g(f)}} & \geq\left[\frac{\bar{\tau}_{f}}{\bar{\tau}_{g}}\right]^{\frac{1}{\lambda_{g}}} \\
\text { i.e., } \bar{\tau}_{g}(f) & \geq\left[\frac{\bar{\tau}_{f}}{\bar{\tau}_{g}}\right]^{\frac{1}{\lambda_{g}}} .
\end{aligned}
$$

Further we obtain from (3.6) and (3.9), for a sequence of values of $r$ tending to infinity that

$$
\begin{gathered}
T_{g}^{-1} T_{f}(r) \geq T_{g}^{-1}\left[\left(\tau_{f}-\varepsilon\right) \cdot r^{\lambda_{f}}\right] \\
\text { i.e., } T_{g}^{-1} T_{f}(r) \geq\left(\frac{\left(\tau_{f}-\varepsilon\right) \cdot r^{\lambda_{f}}}{\left(\tau_{g}-\varepsilon\right)}\right)^{\frac{1}{\lambda_{g}}} \\
\text { i.e., } T_{g}^{-1} T_{f}(r) \geq\left[\frac{\left(\tau_{f}-\varepsilon\right)}{\left(\tau_{g}-\varepsilon\right)}\right]^{\frac{1}{\lambda_{g}}} \cdot r^{\frac{\lambda_{f}}{\lambda_{g}}} \\
\text { i.e., } \frac{T_{g}^{-1} T_{f}(r)}{r^{\frac{\lambda_{f}}{\lambda_{g}}}} \geq\left[\frac{\left(\tau_{f}-\varepsilon\right)}{\left(\tau_{g}-\varepsilon\right)}\right]^{\frac{1}{\lambda_{g}}} .
\end{gathered}
$$

As in view of Lemma 2.1, we get that $\frac{\lambda_{f}}{\lambda_{g}} \geq \lambda_{g}(f)$ and as $\varepsilon(>0)$ is arbitrary, therefore it follows from above that

$$
\begin{aligned}
\limsup _{r \rightarrow \infty} \frac{T_{g}^{-1} T_{f}(r)}{r^{\lambda_{g}(f)}} & \geq\left[\frac{\tau_{f}}{\tau_{g}}\right]^{\frac{1}{\lambda_{g}}} \\
\text { i.e., } \bar{\tau}_{g}(f) & \geq\left[\frac{\tau_{f}}{\tau_{g}}\right]^{\frac{1}{\lambda_{g}}} .
\end{aligned}
$$


On the Measurement of Growth Properties of Entire and Meromorphic Functions 1023

Now from (3.3) and in view of (3.5), we get for a sequence of values of $r$ tending to infinity that

$$
\begin{gathered}
T_{g}^{-1} T_{f}(r) \geq T_{g}^{-1}\left[\left(\sigma_{f}-\varepsilon\right) \cdot r^{\rho_{f}}\right] \\
\text { i.e., } T_{g}^{-1} T_{f}(r) \geq\left(\frac{\left(\sigma_{f}-\varepsilon\right) \cdot r^{\rho_{f}}}{\left(\sigma_{g}+\varepsilon\right)}\right)^{\frac{1}{\rho_{g}}} \\
\text { i.e., } T_{g}^{-1} T_{f}(r) \geq\left[\frac{\left(\sigma_{f}-\varepsilon\right)}{\left(\sigma_{g}+\varepsilon\right)}\right]^{\frac{1}{\rho_{g}}} \cdot r^{\frac{\rho_{f}}{\rho_{g}}} \\
\text { i.e., } \frac{T_{g}^{-1} T_{f}(r)}{r^{\frac{\rho_{f}}{\rho_{g}}}} \geq\left[\frac{\left(\sigma_{f}-\varepsilon\right)}{\left(\sigma_{g}+\varepsilon\right)}\right]^{\frac{1}{\rho_{g}}} .
\end{gathered}
$$

Analogously from (3.2) and in view of (3.5), it follows for a sequence of values of $r$ tending to infinity that

$$
\begin{gathered}
T_{g}^{-1} T_{f}(r) \geq T_{g}^{-1}\left[\left(\bar{\sigma}_{f}-\varepsilon\right) \cdot r^{\rho_{f}}\right] \\
\text { i.e., } T_{g}^{-1} T_{f}(r) \geq\left(\frac{\left(\bar{\sigma}_{f}-\varepsilon\right) \cdot r^{\rho_{f}}}{\left(\bar{\sigma}_{g}+\varepsilon\right)}\right)^{\frac{1}{\rho_{g}}} \\
\text { i.e., } T_{g}^{-1} T_{f}(r) \geq\left[\frac{\left(\bar{\sigma}_{f}-\varepsilon\right)}{\left(\overline{\left.\bar{\sigma}_{g}+\varepsilon\right)}\right]^{\frac{1}{\rho_{g}}} \cdot r^{\frac{\rho_{f}}{\rho_{g}}}}\right. \\
\text { i.e., } \frac{T_{g}^{-1} T_{f}(r)}{r^{\frac{\rho_{f}}{\rho_{g}}}} \geq\left[\frac{\left(\bar{\sigma}_{f}-\varepsilon\right)}{\left(\bar{\sigma}_{g}+\varepsilon\right)}\right]^{\frac{1}{\rho_{g}}} .
\end{gathered}
$$

As in view of Lemma 2.1, $\frac{\rho_{f}}{\rho_{g}} \geq \lambda_{g}(f)$ and as $\varepsilon(>0)$ is arbitrary, we get from (3.9) that

$$
\begin{aligned}
\limsup _{r \rightarrow \infty} \frac{T_{g}^{-1} T_{f}(r)}{r^{\lambda_{g}(f)}} & \geq\left[\frac{\sigma_{f}}{\sigma_{g}}\right]^{\frac{1}{\rho_{g}}} \\
\text { i.e., } \bar{\tau}_{g}(f) & \geq\left[\frac{\sigma_{f}}{\sigma_{g}}\right]^{\frac{1}{\rho_{g}}} .
\end{aligned}
$$

Simialrly, we get from (3.9) that

$$
\begin{aligned}
\limsup _{r \rightarrow \infty} \frac{T_{g}^{-1} T_{f}(r)}{r^{\lambda_{g}(f)}} & \geq\left[\frac{\bar{\sigma}_{f}}{\bar{\sigma}_{g}}\right]^{\frac{1}{\rho_{g}}} \\
\text { i.e., } \bar{\tau}_{g}(f) & \geq\left[\left[\frac{\bar{\sigma}_{f}}{\bar{\sigma}_{g}}\right]^{\frac{1}{\rho_{g}}},\right.
\end{aligned}
$$

since in view of Lemma 2.1, $\frac{\rho_{f}}{\rho_{g}} \leq \lambda_{g}(f)$ and $\varepsilon(>0)$ is arbitrary.

Likewise from (3.3) and in view of (3.9), we get for a sequence of values of $r$ tending to infinity that

$$
T_{g}^{-1} T_{f}(r) \geq T_{g}^{-1}\left[\left(\sigma_{f}-\varepsilon\right) \cdot r^{\rho_{f}}\right]
$$




$$
\begin{aligned}
& \text { i.e., } T_{g}^{-1} T_{f}(r) \geq\left(\frac{\left(\sigma_{f}-\varepsilon\right) \cdot r^{\rho_{f}}}{\left(\bar{\tau}_{g}+\varepsilon\right)}\right)^{\frac{1}{\lambda_{g}}} \\
& \text { i.e., } T_{g}^{-1} T_{f}(r) \geq\left[\frac{\left(\sigma_{f}-\varepsilon\right)}{\left(\overline{\tau_{g}}+\varepsilon\right)}\right]^{\frac{1}{\lambda_{g}}} \cdot r^{\frac{\rho_{f}}{\lambda_{g}}} \\
& \text { i.e., } \frac{T_{g}^{-1} T_{f}(r)}{r^{\frac{\rho_{f}}{\lambda_{g}}}} \geq\left[\frac{\left(\sigma_{f}-\varepsilon\right)}{\left(\bar{\tau}_{g}+\varepsilon\right)}\right]^{\frac{1}{\lambda_{g}}} .
\end{aligned}
$$

Since in view of Lemma 2.1, we get that $\frac{\rho_{f}}{\lambda_{g}} \geq \lambda_{g}(f)$ and as $\varepsilon(>0)$ is arbitrary, therefore it follows from above that

$$
\begin{aligned}
\limsup _{r \rightarrow \infty} \frac{T_{g}^{-1} T_{f}(r)}{r^{\lambda_{g}(f)}} & \geq\left[\frac{\sigma_{f}}{\bar{\tau}_{g}}\right]^{\frac{1}{\lambda_{g}}} \\
\text { i.e., } \bar{\tau}_{g}(f) & \geq\left[\frac{\sigma_{f}}{\bar{\tau}_{g}}\right]^{\frac{1}{\lambda_{g}}} .
\end{aligned}
$$

Further from (3.2) and in view of (3.9), it follows for a sequence of values of $r$ tending to infinity that

$$
\begin{gathered}
T_{g}^{-1} T_{f}(r) \geq T_{g}^{-1}\left[\left(\bar{\sigma}_{f}-\varepsilon\right) \cdot r^{\rho_{f}}\right] \\
\text { i.e., } T_{g}^{-1} T_{f}(r) \geq\left(\frac{\left(\bar{\sigma}_{f}-\varepsilon\right) \cdot r^{\rho_{f}}}{\left(\tau_{g}-\varepsilon\right)}\right)^{\frac{1}{\lambda_{g}}} \\
\text { i.e., } T_{g}^{-1} T_{f}(r) \geq\left[\frac{\left(\bar{\sigma}_{f}-\varepsilon\right)}{\left(\tau_{g}-\varepsilon\right)}\right]^{\frac{1}{\lambda_{g}}} \cdot r^{\frac{\rho_{f}}{\lambda_{g}}} \\
\text { i.e., } \frac{T_{g}^{-1} T_{f}(r)}{r^{\frac{\rho_{f}}{\lambda_{g}}}} \geq\left[\frac{\left(\bar{\sigma}_{f}-\varepsilon\right)}{\left(\tau_{g}-\varepsilon\right)}\right]^{\frac{1}{\lambda_{g}}} .
\end{gathered}
$$

As in view of Lemma 2.1, we get that $\frac{\rho_{f}}{\lambda_{g}} \geq \lambda_{g}(f)$ and also as $\varepsilon(>0)$ is arbitrary, it follows from above that

$$
\begin{aligned}
\limsup _{r \rightarrow \infty} \frac{T_{g}^{-1} T_{f}(r)}{r^{\lambda_{g}(f)}} & \geq\left[\frac{\bar{\sigma}_{f}}{\tau_{g}}\right]^{\frac{1}{\lambda_{g}}} \\
\text { i.e., } \bar{\tau}_{g}(f) & \geq\left[\frac{\bar{\sigma}_{f}}{\bar{\tau}_{g}}\right]^{\frac{1}{\lambda_{g}}} .
\end{aligned}
$$

Again from (3.5) and (3.5), we have for all sufficiently large values of $r$ that

$$
\begin{gathered}
T_{g}^{-1} T_{f}(r) \leq T_{g}^{-1}\left[\left(\bar{\tau}_{f}+\varepsilon\right) \cdot r^{\lambda_{f}}\right] \\
i . e ., T_{g}^{-1} T_{f}(r) \leq\left(\frac{\left(\bar{\tau}_{f}+\varepsilon\right) \cdot r^{\lambda_{f}}}{\left(\bar{\sigma}_{g}-\varepsilon\right)}\right)^{\frac{1}{\rho_{g}}}
\end{gathered}
$$




$$
\begin{aligned}
& \text { i.e., } T_{g}^{-1} T_{f}(r) \leq\left[\frac{\left(\bar{\tau}_{f}+\varepsilon\right)}{\left(\overline{\sigma_{g}}-\varepsilon\right)}\right]^{\frac{1}{\rho_{g}}} \cdot r^{\frac{\lambda_{f}}{\rho_{g}}} \\
& \text { i.e., } \frac{T_{g}^{-1} T_{f}(r)}{r^{\frac{\lambda_{f}}{\rho_{g}}}} \leq\left[\frac{\left(\bar{\tau}_{f}+\varepsilon\right)}{\left(\bar{\sigma}_{g}-\varepsilon\right)}\right]^{\frac{1}{\rho_{g}}} .
\end{aligned}
$$

Since in view of Lemma 2.1, we get that $\frac{\lambda_{f}}{\rho_{g}} \leq \lambda_{g}(f)$ and as $\varepsilon(>0)$ is arbitrary, therefore it follows from above that

$$
\begin{aligned}
\limsup _{r \rightarrow \infty} \frac{T_{g}^{-1} T_{f}(r)}{r^{\lambda_{g}(f)}} & \leq\left[\frac{\bar{\tau}_{f}}{\bar{\sigma}_{g}}\right]^{\frac{1}{\rho_{g}}} \\
\text { i.e., } \bar{\tau}_{g}(f) & \leq\left[\frac{\bar{\tau}_{f}}{\bar{\sigma}_{g}}\right]^{\frac{1}{\rho_{g}}} .
\end{aligned}
$$

Thus the first part of the theorem follows from $(3.9),(3.9),(3.9),(3.9),(3.9),(3.9)$ and (3.9).

Further from (3.6) and in view of (3.9), we get for all sufficiently large values of $r$ that

$$
\begin{gathered}
T_{g}^{-1} T_{f}(r) \geq T_{g}^{-1}\left[\left(\tau_{f}-\varepsilon\right) \cdot r^{\lambda_{f}}\right] \\
\text { i.e., } T_{g}^{-1} T_{f}(r) \geq\left(\frac{\left(\tau_{f}-\varepsilon\right) \cdot r^{\lambda_{f}}}{\left(\bar{\tau}_{g}+\varepsilon\right)}\right)^{\frac{1}{\lambda_{g}}} \\
\text { i.e., } T_{g}^{-1} T_{f}(r) \geq\left[\frac{\left(\tau_{f}-\varepsilon\right)}{\left(\overline{\left.\tau_{g}+\varepsilon\right)}\right]^{\frac{1}{\lambda_{g}}} \cdot r^{\frac{\lambda_{f}}{\lambda_{g}}}}\right. \\
\text { i.e., } \frac{T_{g}^{-1} T_{f}(r)}{r^{\frac{\lambda_{f}}{\lambda_{g}}}} \geq\left[\frac{\left(\tau_{f}-\varepsilon\right)}{\left(\bar{\tau}_{g}+\varepsilon\right)}\right]^{\frac{1}{\lambda_{g}}} .
\end{gathered}
$$

Since in view of Lemma 2.1, we get that $\frac{\lambda_{f}}{\lambda_{g}} \geq \lambda_{g}(f)$ and as $\varepsilon(>0)$ is arbitrary, therefore it follows from above that

$$
\begin{aligned}
\liminf _{r \rightarrow \infty} \frac{T_{g}^{-1} T_{f}(r)}{r^{\lambda_{g}(f)}} & \geq\left[\frac{\tau_{f}}{\bar{\tau}_{g}}\right]^{\frac{1}{\lambda_{g}}} \\
\text { i.e., } \tau_{g}(f) & \geq\left[\frac{\tau_{f}}{\bar{\tau}_{g}}\right]^{\frac{1}{\lambda_{g}}} .
\end{aligned}
$$

Again from (3.2) and in view of (3.5), we get for all sufficiently large values of $r$ that

$$
\begin{gathered}
T_{g}^{-1} T_{f}(r) \geq T_{g}^{-1}\left[\left(\bar{\sigma}_{f}-\varepsilon\right) \cdot r^{\rho_{f}}\right] \\
\text { i.e., } T_{g}^{-1} T_{f}(r) \geq\left(\frac{\left\{\left(\bar{\sigma}_{f}-\varepsilon\right) \cdot r^{\rho_{f}}\right\}}{\left(\sigma_{g}+\varepsilon\right)}\right)^{\frac{1}{\rho_{g}}} \\
\text { i.e., } T_{g}^{-1} T_{f}(r) \geq\left[\frac{\left(\bar{\sigma}_{f}-\varepsilon\right)}{\left(\sigma_{g}+\varepsilon\right)}\right]^{\frac{1}{\rho_{g}}} \cdot r^{\frac{\rho_{f}}{\rho_{g}}} \\
\text { i.e., } \frac{T_{g}^{-1} T_{f}(r)}{r^{\frac{\rho_{f}}{\rho_{g}}}} \geq\left[\frac{\left(\bar{\sigma}_{f}-\varepsilon\right)}{\left(\sigma_{g}+\varepsilon\right)}\right]^{\frac{1}{\rho_{g}}} .
\end{gathered}
$$


As in view of Lemma 2.1, $\frac{\rho_{f}}{\rho_{g}} \geq \lambda_{g}(f)$ and $\varepsilon(>0)$ is arbitrary, we get from (3.9) that

$$
\begin{aligned}
\liminf _{r \rightarrow \infty} \frac{T_{g}^{-1} T_{f}(r)}{r^{\lambda g(f)}} & \geq\left[\frac{\bar{\sigma}_{f}}{\sigma_{g}}\right]^{\frac{1}{\rho_{g}}} \\
\text { i.e., } \tau_{g}(f) & \geq\left[\frac{\bar{\sigma}_{f}}{\sigma_{g}}\right]^{\frac{1}{\rho_{g}}} .
\end{aligned}
$$

Again from (3.2) and in view of (3.9), we get for all sufficiently large values of $r$ that

$$
\begin{gathered}
T_{g}^{-1} T_{f}(r) \geq T_{g}^{-1}\left[\left(\bar{\sigma}_{f}-\varepsilon\right) \cdot r^{\rho_{f}}\right] \\
\text { i.e., } T_{g}^{-1} T_{f}(r) \geq\left(\frac{\left(\bar{\sigma}_{f}-\varepsilon\right) \cdot r^{\rho_{f}}}{\left(\bar{\tau}_{g}+\varepsilon\right)}\right)^{\frac{1}{\lambda_{g}}} \\
\text { i.e., } T_{g}^{-1} T_{f}(r) \geq\left[\frac{\left(\bar{\sigma}_{f}-\varepsilon\right)}{\left(\overline{\left.\tau_{g}+\varepsilon\right)}\right]^{\frac{1}{\lambda_{g}}} \cdot r^{\frac{\rho_{f}}{\lambda_{g}}}}\right. \\
\text { i.e., } \frac{T_{g}^{-1} T_{f}(r)}{r^{\frac{\rho_{f}}{\lambda_{g}}}} \geq\left[\frac{\left(\bar{\sigma}_{f}-\varepsilon\right)}{\left(\bar{\tau}_{g}+\varepsilon\right)}\right]^{\frac{1}{\lambda_{g}}} .
\end{gathered}
$$

Since in view of Lemma 2.1, we get that $\frac{\rho_{f}}{\lambda_{g}} \geq \lambda_{g}(f)$ and as $\varepsilon(>0)$ is arbitrary, therefore it follows from above that

$$
\begin{aligned}
\liminf _{r \rightarrow \infty} \frac{T_{g}^{-1} T_{f}(r)}{r^{\lambda_{g}(f)}} & \geq\left[\frac{\bar{\sigma}_{f}}{\bar{\tau}_{g}}\right]^{\frac{1}{\lambda_{g}}} \\
\text { i.e., } \tau_{g}(f) & \geq\left[\frac{\bar{\sigma}_{f}}{\bar{\tau}_{g}}\right]^{\frac{1}{\lambda_{g}}} .
\end{aligned}
$$

Moreover, we get from (3.5) and (3.5) for a sequence of values of $r$ tending to infinity that

$$
\begin{gathered}
T_{g}^{-1} T_{f}(r) \leq T_{g}^{-1}\left[\left(\bar{\tau}_{f}+\varepsilon\right) \cdot r^{\lambda_{f}}\right] \\
\text { i.e., } T_{g}^{-1} T_{f}(r) \leq\left(\frac{\left(\bar{\tau}_{f}+\varepsilon\right) \cdot r^{\lambda_{f}}}{\left(\sigma_{g}-\varepsilon\right)}\right)^{\frac{1}{\rho_{g}}} \\
\text { i.e., } T_{g}^{-1} T_{f}(r) \leq\left[\frac{\left(\bar{\tau}_{f}+\varepsilon\right)}{\left(\sigma_{g}-\varepsilon\right)}\right]^{\frac{1}{\rho_{g}}} \cdot r^{\frac{\lambda_{f}}{\rho_{g}}} \\
\text { i.e., } \frac{T_{g}^{-1} T_{f}(r)}{r^{\frac{\lambda_{f}}{\rho_{g}}}} \leq\left[\frac{\left(\bar{\tau}_{f}+\varepsilon\right)}{\left(\sigma_{g}-\varepsilon\right)}\right]^{\frac{1}{\rho_{g}}} .
\end{gathered}
$$

As in view of Lemma 2.1, we get that $\frac{\lambda_{f}}{\rho_{g}} \leq \lambda_{g}(f)$ and also as $\varepsilon(>0)$ is arbitrary, it follows from above that

$$
\begin{aligned}
\liminf _{r \rightarrow \infty} \frac{T_{g}^{-1} T_{f}(r)}{r^{\lambda_{g}(f)}} & \leq\left[\frac{\bar{\tau}_{f}}{\sigma_{g}}\right]^{\frac{1}{\rho_{g}}} \\
\text { i.e., } \tau_{g}(f) & \leq\left[\frac{\bar{\tau}_{f}}{\sigma_{g}}\right]^{\frac{1}{\rho_{g}}} .
\end{aligned}
$$


Similarly, from (3.8) and in view of (3.5), it follows for a sequence of values of $r$ tending to infinity that

$$
\begin{gathered}
T_{g}^{-1} T_{f}(r) \leq T_{g}^{-1}\left[\left(\tau_{f}+\varepsilon\right) \cdot r^{\lambda_{f}}\right] \\
\text { i.e., } T_{g}^{-1} T_{f}(r) \leq\left(\frac{\left(\tau_{f}+\varepsilon\right) \cdot r^{\lambda_{f}}}{\left(\bar{\sigma}_{g}-\varepsilon\right)}\right)^{\frac{1}{\rho_{g}}} \\
\text { i.e., } T_{g}^{-1} T_{f}(r) \leq\left[\frac{\left(\tau_{f}+\varepsilon\right)}{\left(\bar{\sigma}_{g}-\varepsilon\right)}\right]^{\frac{1}{\rho_{g}}} \cdot r^{\frac{\lambda_{f}}{\rho_{g}}} \\
\text { i.e., } \frac{T_{g}^{-1} T_{f}(r)}{r^{\frac{\lambda_{f}}{\rho_{g}}}} \leq\left[\frac{\left(\tau_{f}+\varepsilon\right)}{\left(\overline{\left.\sigma_{g}-\varepsilon\right)}\right.}\right]^{\frac{1}{\rho_{g}}} .
\end{gathered}
$$

Since in view of Lemma 2.1, we get that $\frac{\lambda_{f}}{\rho_{g}} \leq \lambda_{g}(f)$ and as $\varepsilon(>0)$ is arbitrary, therefore it follows from above that

$$
\begin{aligned}
\liminf _{r \rightarrow \infty} \frac{T_{g}^{-1} T_{f}(r)}{r^{\lambda_{g}(f)}} & \leq\left[\frac{\tau_{f}}{\bar{\sigma}_{g}}\right]^{\frac{1}{\rho_{g}}} \\
\text { i.e., } \tau_{g}(f) & \leq\left[\frac{\tau_{f}}{\bar{\sigma}_{g}}\right]^{\frac{1}{\rho_{g}}} .
\end{aligned}
$$

Hence the second part of the theorem follows from $(3.9),(3.9),(3.9),(3.9)$ and $(3.9)$.

\section{Acknowledgment}

The authors are thankful to the referee for their valuable suggestions, which considerably improved the presentation of the paper.

\section{REF E R E N C E S}

1. L. Bernal: Crecimiento relativo de funciones enteras. Contribución al estudio de lasfunciones enteras con indice exponencial finito. Doctoral Dissertation, University of Seville, Spain, 1984.

2. L. Bernal: Orden relative de crecimiento de funciones enteras. Collect. Math. 39 (1988), 209-229.

3. L. Debnath, S. K. Datta, T. Biswas and A. Kar: Growth of meromorphic functions depending on $(p, q)$-th relative order. Facta Universititatis series: Mathematics and Informatics, 31(3) (2016), 691-705.

4. S. K. DATTA and A. JHA : On the weak type of meromorphic functions. Int. Math. Forum, 4(12) (2009), 569-579.

5. S. K. DATTA and A. BISWAS : On relative type of entire and meromorphic functions. Advances in Applied Mathematical Analysis, 8(2) (2013), 63-75. 
6. W. K. Hayman: Meromorphic Functions. The Clarendon Press, Oxford 1964.

7. B. K. LAHIRI AND D. BANERJEe : Relative order of entire and meromorphic functions. Proc. Nat. Acad. Sci. India Ser. A. 69(A)(3) (1999), 339-354.

8. E.C. Titchmarsh : The theory of functions. 2nd ed.. Oxford University Press, Oxford , 1968.

9. G. VALiRon : Lectures on the general theory of integral functions. Chelsea Publishing Company, 1949.

Sanjib Kumar Datta

Department of Mathematics

University of Kalyani

Kalyani, Dist-Nadia

Pin-741235, West Bengal, India

sanjib_kr_datta@yahoo.co.in

Tanmay Biswas

Rajbari, Rabindrapalli

R. N. Tagore Road

P.O. Krishnagar, Dist-Nadia

PIN- 741101, West Bengal, India

tanmaybiswas_math@rediffmail.com 